\title{
Coronary Heart Disease and Fish Oil
}

Talya Spivack, $M D$

\section{Introduction}

The benefits of fish consumption on cardiovascular disease has been suggested since the early 1980s in studies that demonstrated Greenland Eskimo's low rates of death from coronary heart disease. ${ }^{1}$ This was followed by another observational study that demonstrated the same findings in Japan. ${ }^{2}$ The proposed linkage between these two groups of people is their propensity to consume fish. Since then many studies have examined the relationship between fish consumption and coronary heart disease (CHD) mortality rates. Current thinking suggests that the primary benefit of consuming fish is secondary to the omega-3 fatty acids found in fish oil. ${ }^{16,17}$

\section{Omega -3 Fatty Acid Sources and Action}

Omega-3 fatty acids are long-chain polyunsaturated fatty acids (18-22 carbon atoms in chain length) with the first of double bonds beginning with the third carbon atom. Plants and marine fish are the two main dietary sources of omega-3 fatty acids found in nature. For the purposes of this article, we will mainly discuss the role of marine omega- 3 fatty acids. These are generally found in oily fish such as mackerel, lake trout, herring, sardines, albacore tuna and salmon. There are two types of marine omega-3 fatty acids: eicosapentaenoic acid (EPA) \& docosahexanoic acid (DHA). In the human body, these are broken down by the enzymes cyclo-oxygenase and then lipoxygenase to create eicosanoids.

The exact mechanism of action of omega-3 fatty acids on CHD remains unknown. However, several ideas have been postulated. Omega-3 fatty acids have been shown to decrease serum triglyceride concentrations in a dose dependent relationship by $25-30 \% .{ }^{10}$ Omega-3 fatty acids have also been linked to decreased platelet aggregation ${ }^{11,12,6}$ as well as the ability to stabilize the myocardium against ventricular arrhythmia in animal studies. ${ }^{13,14,15,6}$

\section{The Evidence}

One of the first significant prospective trials done demonstrating the correlation between consumption of omega- 3 fatty acids and coronary heart disease was done in the Netherlands over a 20 year period. Information about the fish consumption of 852 middle-aged men without coronary heart disease was collected by a careful dietary history obtained from the participants and their wives. During 20 years of follow-up 78 men died from coronary heart disease. An inverse dose-response relation was observed between fish consumption in 1960 and death from coronary heart disease during 20 years of follow-up. Mortality from coronary heart disease was more than $50 \%$ lower among those who consumed at least 30 grams of fish per day than among those who did not eat fish. It was concluded that the consumption of as little as one or two fish dishes per week may be of preventive value in relation to coronary heart disease. ${ }^{3}$

The Netherlands study was shortly followed by the Diet and Reinfarction Trial (DART) of 1989. This was a randomized control trial with factorial design performed with the intent to examine the effect of dietary intervention in secondary prevention of MI. The major endpoints of the study were total mortality and ischemic heart disease events (IHD events and nonfatal MI). Men enrolled in the trial were weighed, measured, and randomly assigned to receive or not receive dietary advice on three factors. The first factor was total fat intake. The men in this arm were designated to reduce fat intake to $30 \%$ of total energy and to increase the polyunsaturated/saturated fat ratio as much as possible. The next arm was the fish arm. Men were told to consume at least two weekly portions (200-400 g) of fatty fish (mackerel, herring, kipper, pilchard, sardine, salmon, and trout). Men who could not tolerate fish were given the option of taking three fish oil capsules per day, providing a total of $900 \mathrm{mg}$ EPA and DHA. The next arm of the study examined fiber intake. These men were instructed to increase their intake of cereal fiber to $18 \mathrm{~g}$ daily. Weight reduction advice and smoking advice were given as seen fit to all arms of the study. The results were surprising. The fish oil arm was shown to have the most CHD benefit demonstrated by an overall reduction of cardiovascular disease mortality by $29 \%$. Surprisingly, the overall dietary fat group did demonstrate a significant decrease in the number of MIs suffered but did not show any overall benefit in mortality. ${ }^{4}$

The next large randomized control study to demonstrate a similar benefit was The Indian Experiment of Infarct Survival published in 1997. This randomized placebo controlled trial aimed to test the effect of fish oil and mustard oil supplementation versus placebo on complications and cardiac events in patients with prior suspected acute myocardial infarction during a follow-up period of 1 year after symptoms were first experienced. Participants were randomized to fish oil (6 capsules per day, providing approximately $2 \mathrm{~g}$ of omega- 3 fatty acids), mustard seed oil (a source of a-linolenic acid, $2.9 \mathrm{~g} / \mathrm{d}$ provided in $20 \mathrm{~mL}$ of oil), and placebo (aluminum hydroxide, $100 \mathrm{mg}$ ). After one year, total cardiac events were $25 \%$ and $28 \%$ in the fish oil and mustard oil groups, respectively, versus $35 \%$ in the placebo group. ${ }^{5,6}$

The success of the DART trial and the Indian Experiment of Infarct Survival trial spawned the Gruppo Italiano per lo Studio della Sopravvivenza nell'Infarto miocardico (GISSI) trial in 1999. This trial was a secondary prevention clinical trial designed to assess the effects of omega-3 polyunsaturated fatty acid and vitamin $\mathrm{E}$ supplementation on mortality and recurrent events in patients with recent MI. The study randomized 11,324 
patients who had survived a myocardial infarction within the last 3 months to four arms: omega-3 fatty acid group received one capsule per day of a highly concentrated product containing about $850 \mathrm{mg}$ of EPA and DHA, the vitamin-E group received $300 \mathrm{mg} / \mathrm{d}$ of synthetic a-tocopherol, the combined treatment group received both, and the control group received neither. The GISSI trial was the first demonstration that a pharmaceutical preparation of omega- 3 fatty acids in addition to dietary and other accepted interventions has a favorable effect on clinical endpoints in post-MI patients. Another unexpected finding was those taking the omega- 3 fatty acids experienced a $20 \%$ reduction in overall mortality and a $45 \%$ decrease in risk for sudden cardiac death. ${ }^{7,8}$

One of the confounders of the above randomized control trials was that the trials had only included men. As a result, a retrospective analysis relating coronary artery disease and fish oil was performed on the cohort of female patients used for the Nurse's Health Study. The end point for this study was incidence of CHD including CHD deaths and nonfatal MI. Women were divided into 5 categories based on frequency of fish consumption: < 1 serving per month, 1-3 servings per month, 1 serving per week, 2-4 servings per week, and 5 servings per week. This trial showed a stepwise decrease in all arms related to more fish consumption in total coronary heart disease cases, fatal coronary heart disease events, and nonfatal MI. ${ }^{9}$ Although this study is convincing, it is also limited by the inability to control for the fact that people who consume more fish products may have an inherently healthier lifestyle.

\section{Conclusions}

The physician must weigh the risks and benefits of prescribing fish oil to their patients. It is important to realize the antiplatelet effects and theoretical ramifications in using omega- 3 fatty acids therapeutically in patients with bleeding tendencies. In addition, pregnant and nursing woman must be cautioned against consuming certain fish such as shark, swordfish, golden bass, and king mackerel that have a higher proportion of mercury. Studies suggest that prenatal mercury exposure can be detrimental to neurological development in children. ${ }^{18,19}$

In 2002, the American Heart Association (AHA) published guidelines advocating the use of omega- 3 fatty acids in patients with and without documented CHD. In patients without CHD they recommended eating a variety of (preferably oily) fish at least twice a week. In patients with $\mathrm{CHD}$ they recommended consuming approximately 1 gram of EPA and DHA daily, preferably from oily fish. In addition they suggested that in this patient population fish oil capsules could be considered in consultation with physicians. In the patients needing triglyceride lowering the AHA recommended $2-4$ grams of EPA and DHA daily provided as capsules under a physicians care. ${ }^{6}$

Omega 3 fatty acids have been shown in epidemiological studies and randomized control studies to decrease the incidence of coronary heart disease both in patients with existing coronary heart disease and prospectively. Although more studies are needed to examine the exact mechanism of action of omega- 3 fatty acids, studies do show a substantial benefit related to the increase of intake of fish oil, both in capsule form and through dietary sources. In conclusion, evidence suggests recommending omega-3 fatty acids to patients at risk for and who have suffered from cardiovascular heart disease.

\section{References}

1. Bang HO, Dyerberg J. Lipid metabolism and ischemic heart disease in Greenland Eskimos. In: Draper H, ed. Advances in Nutrition Research. New York, NY: Plenum Press; 1980:1-22.

2. Keys A. Seven Countries: A Multivariate Analysis of Death and Coronary Heart Disease. Cambridge, Mass: Harvard University Press; 1980

3. Kromhout D, Bosschieter EB, de Lezenne Coulander C. The inverse relation between fish consumption and 20-year mortality from coronary heart disease. $\mathrm{N}$ Engl J Med 1985;312:1205-9.

4. Burr ML, Fehily AM, Gilbert JF, et al. Effects of changes in fat, fish, and fiber intakes on death and myocardial reinfarction: Diet and Reinfarction Trial (DART). Lancet 1989;334:757-61

5. Singh RB, Niaz MA, Sharma JP, Kumar R, Rastogi V, Moshiri M. Randomized, double-blind, placebo-controlled trial of fish oil and mustard oil in patients with suspected acute myocardial infarction: the Indian experiment of infarct survival-4. Cardiovasc Drugs Ther 1997;11:485-91.

6. Penny M. Kris-Etherton, William S. Harris, Lawrence J. Appel and for the Nutrition Fish Consumption, Fish Oil, Omega-3 Fatty Acids, and Cardiovascular Disease. Circulation 2002;106;2747-2757

7. Marchioli R, Barzi F, Bomba E, et al. Early protection against sudden death by n3 polyunsaturated fatty acids after myocardial infarction: time-course analysis of the results of the Gruppo Italiano per lo Studio della Sopravvivenza nell'Infarto Miocardico (GISSI)-Prevenzione. Circulation 2002; 105:1897-903.

8. GISSI Investigators. Dietary supplementation with $\mathrm{n} 3$ polyunsaturated fatty acids and vitamin $\mathrm{E}$ after myocardial infarction: results of the GISSI-Prevenzione trial. Lancet 1999; 354:447-55.

9. Hu FB, Bronner L, Willett WC, et al. Fish and omega-3 fatty acid intake and risk of coronary heart disease in women. JAMA 2002;287:1815-21.

10. Harris WS. n-3 Fatty acids and serum lipoproteins: human studies. Am J Clin Nutr. 1997; 65(5 Suppl):1645S-1654S.

11. Mori TA, Beilin LJ, Burke V, et al. Interactions between dietary fat, fish, and fish oils and their effects on platelet function in men at risk of cardiovascular disease. Arterioscler Thromb Vasc Biol. 1997;17:279-286.

12. Agren JJ, Vaisanen S, Hanninen O, et al. Hemostatic factors and platelet aggregation after a fish-enriched diet or fish oil or docosahexaenoic acid supplementation. Prostaglandins Leukot Essent Fatty Acids. 1997;57:

13. 419-421.Pepe S, McLennan PL. Dietary fish oil confers direct antiarrhythmic properties on the myocardium of rats. J Nutr. 1996;126:34-42.

14. Kinoshita I, Itoh K, Nishida-Nakai M, et al. Antiarrhythmic effects of eicosapentaenoic acid during myocardial infarction-enhanced cardiac microsomal (Ca2_-Mg2_)-ATPase activity. Japan Circ J. 1994;58:903-912.

15. Billman GE, Kang JX, Leaf A. Prevention of ischemia-induced cardiac sudden death by n-3 polyunsaturated fatty acids in dogs. Lipids. 1997;32:1161-1168.

16. Sinclair HM. Deficiency of essential fatty acids and atherosclerosis, etcetera. Lancet 1956; i: 381-3.

17. Dyerberg J, Bang HO, Stofferson E, Moncada S, Vane JR. Eicosapentaenoic acid and prevention of thrombosis and atherosclerosis? Lancet 1978; ii: 117-9.

18. US Environmental Protection Agency. Mercury Study report to Congress. http:// www.epa.gov/mercury/report.htm.

19. Agricultural Research Service, US Department of Agriculture. USDA National Nutrient Database for FISH INTAKE, CONTAMINANTS, AND HUMAN HEALTH $\odot 2006$ American Medical Association. Standard Reference-Release 18 (2005). Washington, DC: US Dept of Agriculture; 2006. 